\title{
Time variations of narrow absorption lines in high resolution quasar spectra ${ }^{\star}$
}

\author{
P. Boissé ${ }^{1,2}$, J. Bergeron ${ }^{1,2}$, J. X. Prochaska ${ }^{3}$, C. Péroux ${ }^{4}$, and D. G. York ${ }^{5}$ \\ ${ }^{1}$ Sorbonne Universités, UPMC Univ. Paris 06, UMR 7095, Institut d'Astrophysique de Paris, 75014 Paris, France \\ e-mail: boisse@iap.fr \\ 2 CNRS, UMR 7095, Institut d'Astrophysique de Paris, 75014 Paris, France \\ 3 UCO/Lick Observatory, UC Santa Cruz, Santa Cruz, CA 95064, USA \\ 4 Aix-Marseille Université, CNRS, LAM (Laboratoire d'Astrophysique de Marseille) UMR 7326, 13388 Marseille, France \\ 5 Department of Astronomy, University of Chicago, 5640 South Ellis Avenue, Chicago, IL 60637, USA
}

Received 9 April 2015 / Accepted 28 May 2015

\begin{abstract}
Aims. We have searched for temporal variations of narrow absorption lines in high resolution quasar spectra. A sample of five distant sources were assembled, for which two spectra are available, either VLT/UVES or Keck/HIRES, which were taken several years apart. Methods. We first investigate under which conditions variations in absorption line profiles can be detected reliably from high resolution spectra and discuss the implications of changes in terms of small-scale structure within the intervening gas or intrinsic origin. The targets selected allow us to investigate the time behaviour of a broad variety of absorption line systems by sampling diverse environments: the vicinity of active nuclei, galaxy halos, molecular-rich galaxy disks associated with damped Ly $\alpha$ systems, as well as neutral gas within our own Galaxy.

Results. Intervening absorption lines from $\mathrm{Mg}$ II, Fe II, or proxy species with lines of lower opacity tracing the same kind of (moderately ionised) gas appear in general to be remarkably stable ( $1 \sigma$ upper limits as low as $10 \%$ for some components on scales in the range 10-100 au), even for systems at $z_{\mathrm{abs}} \approx z_{\mathrm{e}}$. Marginal variations are observed for Mg II lines towards PKS 1229-021 at $z_{\mathrm{abs}}=0.83032$; however, we detect no systems that display any change as large as those reported in low resolution SDSS spectra. The lack of clear variations for low $\beta \mathrm{Mg}$ II systems does not support the existence of a specific population of absorbers made of sweptup gas towards blazars. In neutral or diffuse molecular media, clear changes are seen for Galactic Na I lines towards PKS 1229-02 (decrease in $N$ by a factor of four for one of the five components over $9.7 \mathrm{yr}$ ), corresponding to structure on a scale of about 35 au, in good agreement with known properties of the Galactic interstellar medium. Tentative variations are detected for $\mathrm{H}_{2} J=3$ lines towards FBQS J2340 -0053 at $z_{\text {abs }}=2.05454(\simeq 35 \%$ change in column density, $N$, over $0.7 \mathrm{yr}$ in the rest frame), suggesting there is structure at the 10 au-scale for this warm molecular gas. A marginal change is also seen in C I from another velocity component of the same absorption system $(\simeq 70 \%$ variation in $N(\mathrm{C} \mathrm{I})$.
\end{abstract}

Key words. quasars: absorption lines - ISM: structure

\section{Introduction}

Absorption lines in distant active galactic nuclei (AGN) spectra have long been recognised as an invaluable source of information on diffuse material lying along these lines of sight (LoS), from local interstellar material within the Milky Way, disks or halos of intervening galaxies, intergalactic clouds, up to gas located in the vicinity of the AGN itself. As illustrated by numerous studies (see e.g. Noterdaeme et al. 2007; Neeleman et al. 2015), many properties of the gas can be investigated through a detailed analysis of the absorption lines, including ionisation level, chemical composition, density, and temperature.

Aside from the broad absorption lines (BAL) and intrinsic narrow absorption lines that often display time variations on timescales of months or years in the absorber's frame (Hamann et al. 2008, 2011; Filiz Ak et al. 2013 and references therein; Misawa et al. 2014; Grier et al. 2015), intervening absorption systems observed in quasar spectra are thought to be essentially

* Based on observations with UVES on the Very Large Telescope at the European Southern Observatory (under programs 67.C-0157, 68.A-0170, 074.B-0358, 278.A-5048, 082.A-0569, 087.A-0597, and 087.A-0778) and with HIRES on the Keck Telescope. stable in time. However, a recent study based on multi-epoch low resolution SDSS spectra (Hacker et al. 2013) suggests that some narrow intervening systems (mainly Mg II and Fe II) might also display variability. These authors propose that time changes are due to the transverse motion of the LoS through the absorber coupled to the presence of structure in the absorbing gas at scales in the range 10-100 au, similar to what is observed for diffuse neutral gas in our own Galaxy (Crawford 2003; Lauroesch 2007; Welty 2007; Meyer et al. 2012). Indeed, transverse peculiar velocity values of a few $100 \mathrm{~km} \mathrm{~s}^{-1}$ are expected for the target, the observer, and the intervening gas, which imply drifts over a few years falling in this range. (We recall that $1 \mathrm{au} \mathrm{yr}^{-1}$ is equivalent to $4.67 \mathrm{~km} \mathrm{~s}^{-1}$.)

Information on spatial structure within the low-ionisation medium similar to the one probed by multi-epoch SDSS spectra remains quite limited. Observations of gravitationally lensed quasars with multiple images have been used to investigate spatial variations on scales of a few $100 \mathrm{pc}$. In particular, Rauch et al. (2002) studied the behaviour of Mg II-Fe II systems along three adjacent LoS towards Q2237+0305 and, by comparing the line profiles, infer a lower limit of about $0.5 \mathrm{kpc}$ for the overall absorber extent and a typical size of the order of $100 \mathrm{pc}$ 
for cloudlets associated with individual velocity components. Similarly, towards the lensed quasi-stellar object (QSO) pair APM 08279+5255, Kobayashi et al. (2002) derived an estimate for the cloudlet size of $200 \mathrm{pc}$, in good agreement with values inferred from earlier modelling of these absorbers (Churchill \& Charlton 1999; Crighton et al. 2015). Such observations are restricted to a few systems and do not enable a study of the structure on smaller scales. In our own Galaxy, structure in the lowionisation medium has been investigated by Welty (2007), who found no change in O I, Si II, S II, and Fe II lines over tens of au. Thus, the limited information available (see also the recent study by Neeleman et al. 2015) does not suggest the presence of significant structure on scales as small as 100 au in the low-ionisation medium, comparable to the one seen in the neutral medium.

The results obtained by Hacker et al. (2013) then appear somewhat puzzling and require additional investigations. Their study is based on low resolution SDSS spectra, which can only provide equivalent width measurements and do not give access to the true line profiles and to the identification of the individual absorption velocity components. If some absorption lines do vary, changes most probably affect only some velocity components and should be much more apparent in high resolution spectra. High resolution line profiles would also be very useful for assessing the reality of any variation detection. Indeed, when spectral profiles are resolved (or at least partially resolved), changes in a specific transition from a given species imply a corresponding change for other transitions from the same species that can be computed a priori, providing a stringent reliability test. Furthermore, high resolution data will minimise the impact of various artefacts like imperfect sky subtraction, bad pixels, etc.

Good signal-to-noise $(\mathrm{S} / \mathrm{N})$ high resolution quasar spectra have been taken routinely over the past $15 \mathrm{yr}$ (for instance, in the context of Large Programs conducted on 8-10 m class telescopes; see e.g. Bergeron et al. 2004; Molaro et al. 2013) and for some distant sources, several observations have been performed over time intervals reaching ten years. We have thus gathered a sample of five sources with at least two VLT/UVES or Keck/HIRES spectra taken several years apart. In one case, we acquired a spectrum specifically for this study. These LoS all together include a broad variety of systems, probing various environments ranging from neutral Galactic gas, galaxy disks at intermediate redshifts (i.e. damped Ly $\alpha$ system), Mg II-Fe II systems due to intervening galaxy halos, as well as systems with a redshift close to the emission redshift. Several of our targets fall in the blazar category, which will allow us to investigate the possible presence of narrow systems with a high ejection velocity, in line with the scenario put forward by Bergeron et al. (2011).

The purpose of our study is to explore the potential of multiepoch high-resolution observations of AGN to investigate the small-scale structure in intervening gas and possible intrinsic variations for narrow low ejection velocity systems. In Sect. 2, we describe the method (expected LoS drifts, selection of transitions that are best suited for a search of variations, etc.) while the data and raw results are presented in Sect. 3. Their implications are discussed in Sect. 4. We summarise our results and mention some prospects for further variability studies in Sect. 5 .

\section{Detecting absorption line variations}

When considering two spectra of the same target taken over some time interval, an important parameter is the drift of the LoS relative to a foreground absorber. We first discuss how one can estimate this scalelength, over which the comparison of the

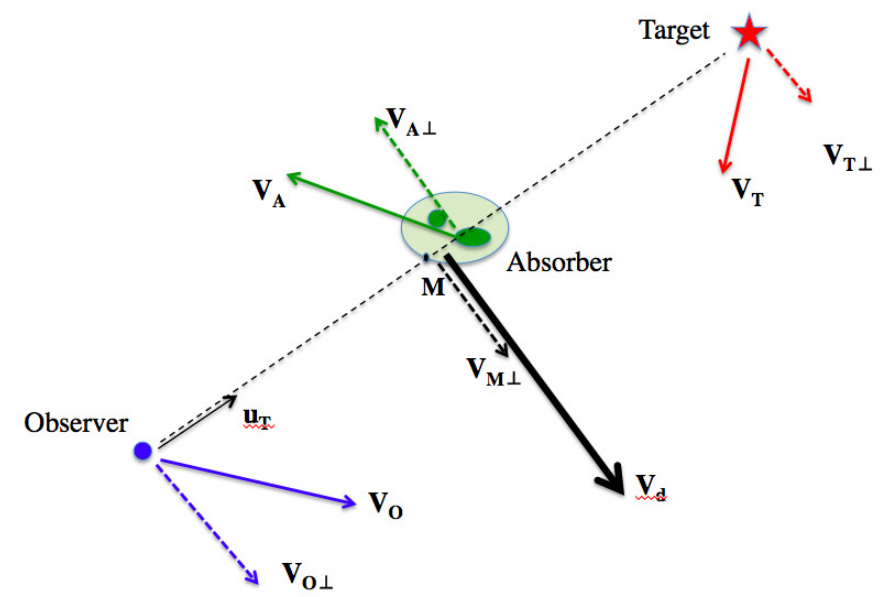

Fig. 1. Observer and target move with $3 \mathrm{D}$ velocities $\boldsymbol{V}_{\mathrm{O}}$ and $\boldsymbol{V}_{\mathrm{T}}$ in the cosmological frame. Their transverse component $\boldsymbol{V}_{\mathrm{O} \perp}$ and $\boldsymbol{V}_{\mathrm{T} \perp}$ determines the transverse velocity $\boldsymbol{V}_{\mathrm{M} \perp}$ of the impact point $\mathrm{M}$. The latter combines with the transverse velocity of the absorber $\boldsymbol{V}_{\mathrm{A} \perp}$ to define the relative drift velocity of the LoS with respect to the gas, $\boldsymbol{V}_{\mathrm{d}}$, which sets the scale probed within the absorber during a given time interval.

two observations will provide structure information. Next, we examine which line parameters (the opacity in particular) yield the best sensitivity to a given fractional column density change. Finally, we discuss methods that can be used to reliably detect line variations and assess their significance.

\subsection{Line of sight drifts relative to the absorber}

For now, we consider that the target is point-like and that it defines an "ideal" LoS, intersecting an intermediate-redshift absorber at point M (hereafter the "impact point"; Fig. 1). As mentioned above, it is the combination of small-scale density or velocity structure in the intervening gas and the transverse motion of the LoS through this material that can potentially induce line variations. The appropriate frame for computing the velocities of interest is a cosmological one, in which the cosmic microwave background (CMB) is globally isotropic. The motion of both the target and the observer determines the drift of the LoS in this frame. The observer's motion is due mainly to the peculiar motion of the Milky Way and to Galactic rotation. The combination of the two corresponding velocities is directly constrained by the detection of the dipolar anisotropy in the CMB radiation, indicating that the solar system is moving at $368 \pm 2 \mathrm{~km} \mathrm{~s}^{-1}$ relative to the observable Universe towards the direction $l_{\mathrm{o}}=263.85 \mathrm{deg}$ and $b_{\mathrm{o}}=48.25 \mathrm{deg}$ (Fixsen et al. 1996).

We denote by $\boldsymbol{V}_{\mathrm{O}}$ the corresponding velocity vector. Consider a target $T$ with Galactic coordinates $\left(l_{\mathrm{T}}, b_{\mathrm{T}}\right)$ and let $\boldsymbol{u}_{\mathrm{T}}$ be the unit vector pointing in this direction (Fig. 1). We are only concerned by the transverse component, thus by the projection, $\boldsymbol{V}_{\mathrm{O} \perp}$, of $\boldsymbol{V}_{\mathrm{O}}$ onto a plane normal to $\boldsymbol{u}_{\mathrm{T}}$.

This projected velocity can be written as

$\boldsymbol{V}_{\mathrm{O} \perp}=\boldsymbol{V}_{\mathrm{O}}-\left(\boldsymbol{u}_{\mathrm{T}} \cdot \boldsymbol{V}_{\mathrm{O}}\right) \boldsymbol{u}_{\mathrm{T}}=V_{\mathrm{O}}\left(\boldsymbol{u}_{\mathrm{O}}-\left(\boldsymbol{u}_{\mathrm{T}} \cdot \boldsymbol{u}_{\mathrm{O}}\right) \boldsymbol{u}_{\mathrm{T}}\right)$,

where $\boldsymbol{V}_{\mathrm{O}}=V_{\mathrm{O}} \cdot \boldsymbol{u}_{\mathrm{O}}$. From these relations, and using the components $\left(\cos b_{\mathrm{T}} \cos l_{\mathrm{T}}, \cos b_{\mathrm{T}} \sin l_{\mathrm{T}}, \sin b_{\mathrm{T}}\right)$ of the unit vector associated with Galactic coordinates $\left(l_{\mathrm{T}}, b_{\mathrm{T}}\right)$, it is straightforward to compute $V_{\mathrm{O}, \perp}$ for any specific target (for the five targets listed in Table $1, V_{\mathrm{O}, \perp}=288,368,129,365$, and $12 \mathrm{~km} \mathrm{~s}^{-1}$, respectively). In the most favourable case for which $V_{\mathrm{O}, \perp}=V_{\mathrm{O}}$, this motion 
Table 1. The sample.

\begin{tabular}{lccccccc}
\hline \hline $\begin{array}{l}\text { Target } \\
\text { common name }\end{array}$ & Class & $\begin{array}{c}\text { Coordinates } \\
\text { J2000 }\end{array}$ & $z_{\text {em }}$ & Spectrograph & Date & $\begin{array}{c}\lambda \text { range } \\
\text { nm }\end{array}$ & $\begin{array}{c}\Delta t, n \\
\text { min }\end{array}$ \\
\hline AO 0235+164 & BL- $\gamma$ & $023838.9+163659$ & 0.937 & HIRES & $12-1997$ & $367-612$ & 40,4 \\
& & & & UVES & $02-2007$ & $303-388+58-668$ & 60,4 \\
\hline PKS 0458-020 & FS- $\gamma$ & $050112.8-015914$ & 2.286 & HIRES & $02-1995$ & $394-629$ & $480^{b}$ \\
& & & & UVES & $10-2004$ & $390-665+670-850$ & 75,4 \\
\hline PKS 1229-02 & FS- $\gamma$ & $123200.0-022404$ & 1.044 & UVES & $02-2002$ & $326-445+458-668$ & 60,3 \\
& & & & UVES & $04-2011$ & $326-445+458-668$ & 50,1 \\
\hline PKS 1741-038 & FS- $\gamma$ & $174358.8-035004$ & 1.054 & UVES & $06-2001$ & $414-621$ & 40,2 \\
& & & & UVES & $04-2011$ & $414-621$ & 50,5 \\
\hline FBQS J2340-0053 & FS 234023.7-005327 & 2.085 & HIRES & $08-2006$ & $306-589$ & $250^{b}$ \\
& & & & UVES & $10-2008$ & $330-450+478-681$ & 75,6 \\
\hline
\end{tabular}

Notes. Class - BL: BL Lac object, FS: Flat Spectrum Radio QSO (FSRQ), $\gamma$ : Gamma-ray emitter. The four $\gamma$-ray emitters are blazars. Spectrograph - spectral resolution: $F W H M($ HIRES $)=6.25 \mathrm{~km} \mathrm{~s}^{-1}, F W H M($ UVES $)=6.6 \mathrm{~km} \mathrm{~s}^{-1} . \Delta t, n$ Exposure time per individual exposure and number of exposures. ${ }^{(a)}$ In a very high state at both epochs. The new $z_{\mathrm{em}}$ is derived from [Ne IV] emission. ${ }^{(b)}$ Total exposure time.

alone results in a shift of about $4 \times 10^{-3}$ pc or 800 au near the observer, over a time interval of ten years.

Unfortunately, we cannot estimate the transverse velocity of the target because the proper motion onto the sky is much too small to be measurable, contrary to interstellar medium studies, in which the proper motion of nearby bright stars is known. Unless the target belongs to a peculiar environment like a galaxy cluster or has experienced an ejection event, the magnitude of its peculiar velocity should be also a few $100 \mathrm{~km} \mathrm{~s}^{-1}$. The transverse velocity of the impact point $\mathrm{M}$ can then be written as

$\boldsymbol{V}_{\mathrm{M} \perp}=\alpha \boldsymbol{V}_{\mathrm{T} \perp}+(1-\alpha) \boldsymbol{V}_{\mathrm{O} \perp}$

where $\alpha=d_{\mathrm{A}} / d_{\mathrm{T}}, d_{\mathrm{A}}$ as the distance to the absorber and $d_{\mathrm{T}}$ the distance to the target. Finally, one must take the velocity $\boldsymbol{V}_{\mathrm{A}}$ of the absorber into account in the cosmological frame or rather its transverse component, $V_{\mathrm{A} \perp}$. In the end, the value of interest is the relative drift velocity

$\boldsymbol{V}_{\mathrm{d}}=\boldsymbol{V}_{M \perp}-\boldsymbol{V}_{\mathrm{A} \perp}$.

Again, $\boldsymbol{V}_{\mathrm{A} \perp}$ cannot be determined (interstellar studies suffer from the same difficulty), and only a typical value of a few hundred $\mathrm{km} \mathrm{s}^{-1}$ can be considered. As with the observer, the actual value results from the combination of the bulk intervening galaxy motion and of the absorbing gas internal kinematics, e.g. rotation, infall, or outflow. A maximum value for the drift velocity $V_{\mathrm{d}}$ can be estimated assuming an optimal configuration in which $\boldsymbol{V}_{\mathrm{O} \perp}$ and $\boldsymbol{V}_{\mathrm{T} \perp}$ are parallel, while $\boldsymbol{V}_{\mathrm{A} \perp}$ points in the opposite direction. In that case, $V_{\mathrm{d}}$ can reach values $V_{\mathrm{d}}=$ $\max \left(V_{\mathrm{O} \perp}, V_{\mathrm{T} \perp}\right)+V_{\mathrm{A} \perp}$, as large as about $500 \mathrm{~km} \mathrm{~s}^{-1}$.

Equations (2) and (3) are valid in the local 3D space. On large cosmological scales, there is no unique way to define distances. Thus, these relations can hold only at low redshifts where all cosmological distances become equivalent. Since the exact values of both $V_{\mathrm{T} \perp}$ and $V_{\mathrm{A} \perp}$ are unknown for any specific target, it is sufficient for our purpose to estimate drift velocities by i) inserting typical values for $V_{\mathrm{T} \perp}$ and $V_{\mathrm{A} \perp}$ into the above equations; ii) using the angular size distance for $d$; and iii) taking time dilation into account: a time interval $\Delta t$ in the observer's frame corresponds to $\Delta t /\left(1+z_{\mathrm{A}}\right)$ or $\Delta t /\left(1+z_{\mathrm{T}}\right)$ in the absorber's or target's frame respectively, which is equivalent to reduce $V_{\mathrm{A} \perp}$ and $V_{\mathrm{T} \perp}$ by a factor of $\left(1+z_{\mathrm{A}}\right)$ or $\left(1+z_{\mathrm{T}}\right)$.

In fact, a quasar is an extended continuum source with a typical size in the range 10-100 au (the size of the accretion disk responsible for the optical emission; Dai et al. 2010). Thus, unlike observations of moving stars used to probe interstellar gas structure, significant averaging of the foreground structure can occur, implying a reduced amplitude for absorption line variations. The situation may be different for blazars. These sources undergo spectacular photometric variations and, when in a "high state", their continuum is dominated by emission from the relativistic jet. Changes in the morphology of the source itself can also occur, which would be equivalent to an additional contribution to the drift of the LoS. Time changes in the $21 \mathrm{~cm}$ absorption seen towards AO $0235+164$ and PKS $1127-14$ by Wolfe et al. (1982) and Kanekar \& Chengalur (2001) are attributed precisely to such an effect.

When dealing with absorption by Galactic interstellar material (four out of our five selected targets display relatively strong $\mathrm{Ca}$ II or Na I absorption from Milky Way gas), the appropriate frame to consider is the local standard of rest (LSR), in which interstellar gas is at rest on average. In this case, the drift of the LoS is determined by the peculiar motion of the Sun in this frame, $V_{S}$, or more specifically, by its projection onto a plane perpendicular to the LoS considered. This question is discussed in more detail in Boissé et al. (2013).

\subsection{Sensitivity to absorption line variations}

To select the absorption lines that are best suited to searching for time variations, we now quantify the sensitivity to a given fractional column density $(N)$ change. Obviously, very weak or, in contrast, saturated features will be of little use: there must be some optimal intermediate value for the opacity.

Consider a fully resolved normalised profile, $I_{\mathrm{n}}(v)$, expressed in the velocity scale,

$I_{\mathrm{n}}(v)=\mathrm{e}^{-\tau(v)}$,

where $\tau(v)$ is the line opacity at velocity $v$, written as

$\tau(v)=k \lambda_{0} f N(v)$,

where $k$ is a constant, $\lambda_{0}$ the rest wavelength of the transition, $f$ the oscillator strength, and $N(v) \mathrm{d} v$ the column density for species within the velocity interval $[v, v+\mathrm{d} v]$. Let us first assume a spectrum noise that is independent of $I_{\mathrm{n}}$. The sensitivity can then be expressed as

$\left|\frac{\delta I_{\mathrm{n}}}{\delta N / N}\right|=\tau \mathrm{e}^{-\tau}$. 
It reaches its maximum at $\tau=1$, for which $I_{\mathrm{n}} \approx 0.37$. The interval over which the sensitivity is reduced by less than $20 \%$ is $0.61 \leq \tau \leq 1.53$, which corresponds to $0.22 \leq I_{\mathrm{n}} \leq 0.54$.

An alternative case of interest is that of photon noise, for which the spectrum rms follows a $\sqrt{I_{\mathrm{n}}}$ law. The ability to detect a given variation $\delta I_{\mathrm{n}}$ is then proportional to

$\left|\frac{\delta I_{\mathrm{n}}}{\sqrt{I_{\mathrm{n}}} \delta N / N}\right|=\tau \mathrm{e}^{-\frac{\tau}{2}}$

This expression reaches its maximum at $\tau=2$, where $I_{\mathrm{n}} \approx 0.14$ (the sensitivity remains good in the sense defined above for $1.2 \leq \tau \leq 3.1)$. In practice, observed spectra are within these two extreme cases, so we conclude that the sensitivity for the detection of variations is optimal for intermediate opacity values (typically 1.5), corresponding to normalised intensities of about 0.2 .

One should keep in mind that, depending on the instrumental resolution and Doppler $b$ parameter value, absorption profiles will generally not be fully resolved, implying apparent opacities that are lower than the true ones (Savage \& Sembach 1991). For instance, with an instrumental resolution of $6.6 \mathrm{~km} \mathrm{~s}^{-1}$, the depth of a Gaussian line is reduced by a factor of 0.45 for $b=2 \mathrm{~km} \mathrm{~s}^{-1}$. Then, if the true opacity at line centre is $\tau_{0}=1$, the apparent opacity becomes 0.33 . Therefore, to conclude on the sensitivity of a given line to changes in $N$, one cannot rely only on its observed profile. Fortunately, several transitions from the same species are often available with various $f$ values. Simultaneous fitting of the corresponding line profiles will then allow us to determine the true opacity and assess whether these transitions are appropriate for a variability search.

\subsection{Characterisation of line variations}

Our aim is to detect line profile variations and to quantify their significance. A first direct indication can be obtained by superposing the two successive spectra after careful normalisation and adjustment of the wavelength scales if necessary. Such a raw comparison is meaningful only if the spectral resolution, $R$, is the same at the two epochs. If this is not the case, the higher $R$ spectrum has to be degraded to the lower resolution value. Fortunately, the HIRES and UVES data used in this paper have very similar nominal resolutions $-F W H M=6.25$ and $6.6 \mathrm{~km} \mathrm{~s}^{-1}$, respectively, and we could verify that, given the $\mathrm{S} / \mathrm{N}$, such a slight difference has a negligible effect on the profile of the absorption features discussed in this paper (cf. Sect. 3). Differences in the line spread function (LSF) might also play a role. One can use unresolved lines to check that such an effect does not affect the comparison of successive profiles.

It turns out that for the systems investigated here, the two successive spectra available rarely display marked, unambiguously significant differences. Thus, in order to i) increase our ability to detect weak variations by relying on the presence of several transitions from a given species (e.g. Fe II, or $\mathrm{H}_{2}$ ); and ii) take differing $R$ values into account if necessary, a more powerful method is to simultaneously fit all profiles corresponding to these transitions at each epoch. To this purpose, we used VPFIT ${ }^{1}$ a routine that uses multi-component Voigt profiles (convolved with the instrument profile, which we assume to be Gaussian here) and that yields the redshift $z_{i}$, column density $N_{i}$, and Doppler parameter $b_{i}$ for each velocity component, together with their $1 \sigma$ uncertainties. Comparison of the values obtained for each epoch then allows us to determine whether some of these

\footnotetext{
1 http://www.ast.cam.ac.uk/ rfc/vpfit.html
}

parameters have undergone significant time changes. In all cases, the same sets of components were appropriate for fitting the profiles at both epochs with no significant change in $z_{i}$ or in $b_{i}$.

Some time variations in $N_{i}$ are detected or suspected for a few absorptions systems (see Sect. 3). To assess their significance, we computed the difference between epoch 1 and 2 , $\Delta N_{i}=N_{i, 2}-N_{i, 1}$ and its uncertainty $\sigma\left(\Delta N_{i}\right)$ by combining uncertainties on $N_{i, 1}$ and $N_{i, 2}$ in quadrature. This procedure provides a formal significance that properly takes the noise level in both spectra into account. Other sources of uncertainty related to continuum placement or to the choice of velocity components are more difficult to quantify, especially when variations are small. As a conservative approach, variations with a significance level above $3.5 \sigma$ and an associated relative column density variation, $\Delta N /\langle N\rangle$, above $25 \%$ are qualified as "significant". Variations at more than $2 \sigma$ but that do not fulfil these two criteria are considered as "marginal".

When several transitions from the same species are available, the quality of the fit obtained provides a stringent test of the internal consistency of the data and gives a powerful way to rule out false variations due to instrumental artefacts. Indeed, changes must not only be present for all transitions but their magnitude must also be consistent with the $f$ values. In this regard, species such as $\mathrm{Fe}$ II, $\mathrm{C}$ I, or $\mathrm{H}_{2}$, which display many transitions from a given level within a relatively narrow wavelength interval, are especially suitable for probing weakly ionised, neutral, or molecular gas, respectively.

Often, several species can be used to trace a given phase (e.g. $\mathrm{Mg}$ II, Fe II, S II for weakly ionised gas). One can then select among all lines detected from these species those i) whose opacity falls in the optimal range discussed above; and ii) that occur in spectral regions of good $\mathrm{S} / \mathrm{N}$.

\section{Variability study of five blazars with low-moderate $\beta$ systems}

\subsection{The absorber sample}

One of our initial motivations was to explore the link between gas swept by the AGN jet and low $\beta$ absorbers, as suggested by Bergeron et al. (2011). We then investigated among their blazar sample objects with jets, thus radio-loud and $\gamma$-ray emitters (detected by Egret, Integral or Fermi/LAT). An additional selection criterion is the existence of $\mathrm{Mg}$ II absorption systems at low relative velocity $\beta \equiv \Delta v\left(z_{\mathrm{em}}, z_{\mathrm{abs}}\right) / c<0.15$ with a restframe equivalent width $W_{\mathrm{r}}(2796)>0.3 \AA$, as well as intervening systems and Damped Ly- $\alpha$ (DLA) systems at high $\beta$. We then searched the VLT-UVES and Keck-HIRES archives for highspectral resolution spectra of this blazar subsample, and selected targets observed several years apart, possibly about one decade. We checked that the $\mathrm{S} / \mathrm{N}$ per pixel was $S / N \gtrsim 10$ for the main absorptions of interest: the $\mathrm{Mg}$ II doublets at $\beta<0.15$ and the Galactic Na I doublets.

One blazar satisfying the above selection criteria, is the extensively studied, highly variable BL Lac object AO 0235+164 for which variations of $21 \mathrm{~cm}$ intervening absorption at $z_{\mathrm{abs}}=$ 0.524 had been reported three decades ago (Wolfe et al. 1982). This absorber has been identified as a BAL quasar with very faint extended emission (Burbidge et al. 1996). The variations were interpreted as most probably due to variations in the brightness distribution of the background radio source. Another blazar is PKS 1741-038, which was re-observed by us with UVES in 2011 to search for variability of its two strong $\mathrm{Mg}$ II absorbers at $\beta=0.074$ and 0.287 . For both objects, the available UVES 
Table 2. Absorption systems.

\begin{tabular}{|c|c|c|c|c|c|c|}
\hline Target & $z_{\mathrm{abs}}$ & $\beta$ & $\begin{array}{r}\text { Main } \\
\text { doublet }\end{array}$ & Strength & $S / N^{a}$ & $\begin{array}{l}\text { Associated low ions } \\
\text { present in the } 2 \text { spectra }\end{array}$ \\
\hline \multirow[t]{5}{*}{ AO $0235+164$} & +0.00002 & & $\mathrm{NaI}$ & $\mathrm{w}$ & $67-56$ & none \\
\hline & 0.52432 & 0.235 & Mg II & VSm & $28-14^{b}$ & Mg I, Fe II, Mn II, Ca II, Ti II \\
\hline & 0.85142 & 0.045 & Mg II & $\mathrm{w}$ & $62-50$ & none \\
\hline & 0.85237 & 0.045 & $\mathrm{Mg}$ II & S & $62-50$ & $\mathrm{Mg}$ I, Fe II \\
\hline & 0.85562 & 0.043 & Mg II & $\mathrm{w}$ & $62-50$ & none \\
\hline \multirow[t]{4}{*}{ PKS 0458-020 } & 0.89025 & 0.503 & Mg II & $\mathrm{S}$ & $11-13$ & Fe II \\
\hline & 1.52712 & 0.257 & $\mathrm{Mg}$ II & VSm & $15^{b}-5$ & $\mathrm{Fe}$ II \\
\hline & 1.56055 & 0.244 & Mg II & $\mathrm{S}$ & $14^{b}-5$ & C I, Fe II, Zn II, Cr II, Ni II \\
\hline & 2.03956 & 0.078 & $\mathrm{Mg}$ II & S & $10^{b^{\prime}}-5$ & Fe II, Zn II, Cr II, Ni II, Si II \\
\hline \multirow[t]{8}{*}{ PKS 1229-02 } & -0.00016 & & $\mathrm{NaI}$ & $\mathrm{W}$ & $52-15$ & Ca II \\
\hline & 0.39458 & 0.364 & Mg II & VSm & $44-13$ & Mg I, Fe II, Mn II, Ca II, Ti II \\
\hline & 0.69990 & 0.182 & Mg II & Vw & $50-13$ & none \\
\hline & 0.75643 & 0.150 & Mg II & ws & $50-13$ & Mg I, Fe II \\
\hline & 0.76836 & 0.144 & Mg II & W & $50-14$ & none \\
\hline & 0.83032 & 0.110 & Mg II & $\mathrm{w}$ & $53-14$ & Fe II \\
\hline & 0.83102 & 0.110 & Mg II & Vw & $53-14$ & none \\
\hline & 0.83128 & 0.109 & Mg II & w & $53-14$ & Fe II \\
\hline \multirow[t]{6}{*}{ PKS 1741-038 } & -0.00070 & & $\mathrm{NaI}$ & $\mathrm{w}$ & $12-32$ & none \\
\hline & -0.00033 & & $\mathrm{NaI}$ & $\mathrm{S}$ & $12-32$ & DIBs \\
\hline & 0.52739 & 0.288 & Mg II & $\mathrm{w}$ & $5-13$ & none \\
\hline & 0.52822 & 0.287 & Mg II & $\mathrm{S}$ & $5-13$ & $\mathrm{Mg}$ I, Fe II \\
\hline & 0.67846 & 0.199 & Mg II & $\mathrm{w}$ & $8-18$ & none \\
\hline & 0.90799 & 0.074 & Mg II & VSm & $10-28$ & $\mathrm{Mg}$ I, Fe II \\
\hline \multirow[t]{3}{*}{ FBQS J2340-0053 } & -0.00002 & & $\mathrm{NaI}$ & $\mathrm{S}$ & $29-68$ & Ca II \\
\hline & 1.36064 & 0.261 & Mg II & VSm & $30^{b}-64$ & C I, Fe II, Zn II, Cr II, Si II, Ni II \\
\hline & 2.05454 & 0.010 & Fe II & VSm & $34^{b^{\prime}}-56^{b^{\prime}}$ & C I, C I^, Fe II, S II, Si II \\
\hline
\end{tabular}

Notes. ${ }^{(a)} \mathrm{S} / \mathrm{N}$ per pixel around the main absorption for the older and recent epoch, respectively. ${ }^{\left(b, b^{\prime}\right)} \mathrm{Mg}$ II region not covered; we list instead the S/N around Fe II2374-2382, Fe II1608-1611. VSm: Very strong, highly multiple absorption, $w_{\mathrm{r}}(2796) \gtrsim 2.0 \AA$ A S: Strong absorption, $w_{\mathrm{r}}(2796$ or 5891) $>0.3 \AA$. w: Weak absorption, $0.02<w_{\mathrm{r}}(2796$ or 5891$)<0.3 \AA$. ws: Although weak, the Mg II doublet is saturated. Vw: Very weak absorption, $w_{\mathrm{r}}(2796)<0.02 \AA$.

and HIRES spectra have been obtained at epochs about $9.5 \mathrm{yr}$ apart in the observer's frame.

We also searched for high resolution spectra of PKS 1229-02, a blazar for which we obtained deep HST imaging showing optical emission associated with knots in the radio jet (Le Brun et al. 1997). There is a DLA system at $z_{\text {abs }}=0.3950$ with associated $21 \mathrm{~cm}$ absorption, and several $\mathrm{Mg}$ II absorbers at $\beta \leq 0.15$. The above selection criteria are also met for this source and there are two sets of high S/N UVES spectra taken at epochs $9.2 \mathrm{yr}$ apart.

Finally, there are HIRES and UVES data available for two targets with C I absorption at $z_{\text {abs }} \sim 1.5$ with associated $21 \mathrm{~cm}$ absorption at high $\beta$ (Kanekar et al. 2010): PKS 0458-020 $\left(z_{\mathrm{abs}}=1.5606\right)$ and FBQS J2340-0053 $\left(z_{\mathrm{abs}}=1.3608\right)$, the latter with observations about two years apart. In the radio spectrum of PKS 0458-020, variable $21 \mathrm{~cm}$ absorption on timescale of months has been detected at $z_{\mathrm{abs}}=2.0395$ (Kanekar et al. 2014, and references therein). There are also DLA systems at low $\beta \leq 0.10$ toward PKS 0458-020 at $z_{\text {abs }}=2.0395$ and FBQS J2340-0053 at $z_{\text {abs }}=2.0545$. The physical conditions in the low $\beta$ DLA system of FBQS J2340-0053, with associated CI absorption, have been investigated by Jorgenson et al. (2010). The multiple system at $z_{\mathrm{abs}}=1.3608$ toward FBQS J2340-0053 has been extensively studied by Rahmani et al. (2012) to constrain the variation of fundamental constants.

Information on the sample and the observations is given in Table 1 and that on the absorption systems in Table 2. For some UVES data, we used the reduced data sets produced internally at ESO, based on the latest calibration pipelines and the best available calibration data. For the other UVES observations, the spectra were reduced by us or made available to us by several colleagues (see the acknowledgements). For HIRES spectra, the data sets were reduced by Prochaska's team. All redshifts and velocities considered in the following are vacuum heliocentric.

In the analysis presented below, we estimate the variation of $N$ between the two epochs and use the combined quadratic errors to evaluate its significance level, as described in Sect. 2.3. In case of no variation, we give the $1 \sigma$ limit on $\Delta N /\langle N\rangle$, restricting this evaluation to fairly good $\mathrm{S} / \mathrm{N}$, thus meaningful limits: $\Delta N /\langle N\rangle<50 \%$.

\subsection{Tracers of the interstellar medium}

The main tracers of the local interstellar medium (ISM) detectable in our spectra are the $\mathrm{Na}$ I and Ca II doublets, Ca I $\lambda 4227$, as well as the $\mathrm{CH}^{+} \lambda 4232$ and $\mathrm{CH} \lambda 4300$ molecular lines. Diffuse interstellar bands (DIBs), mainly at 4428, 5780, and $5797 \AA$, could also be identified in some UVES spectra.

Towards AO $0235+164$, the Galactic Na I absorption shows a strong, single component at $z_{\mathrm{abs}}=+0.00002$. A simultaneous fit to both members of the NaI doublet yields identical values at the two epochs, $9.2 \mathrm{yr}$ apart, within the $1 \sigma$ errors: $N(\mathrm{NaI})=$ $(2.51 \pm 0.04) \times 10^{12} \mathrm{~cm}^{-2}$.

The Galactic Na I absorption towards PKS 1229-02 is multiple (5 components) and extends over $76 \mathrm{~km} \mathrm{~s}^{-1}$. Significant variation is present for the component at $z_{\mathrm{abs}}=-0.000127$ : in $9.7 \mathrm{yr}$, 
Table 3. Targets with absorption systems showing marginal or significant variability.

\begin{tabular}{|c|c|c|c|c|c|}
\hline Target & $z_{\mathrm{abs}}^{+}$ & Date & $\begin{array}{c}N \\
\mathrm{~cm}^{-2} \\
\end{array}$ & Date & $\begin{array}{c}N \\
\mathrm{~cm}^{-2} \\
\end{array}$ \\
\hline AO $0235+164$ & $\begin{array}{l}\mathrm{Mg} \text { II } \\
0.85232 \\
0.85237^{v} \\
\mathrm{Mg} \mathrm{II} \\
0.85562 \\
\end{array}$ & $\begin{array}{l}\text { (blend) } \\
12-1997 \\
12-1997 \\
12-1997\end{array}$ & $\begin{array}{c}\text { marginal variability }^{a} \\
(2.79 \pm 0.46) \times 10^{12} \\
(5.58 \pm 0.85) \times 10^{12} \\
\text { no variability } \\
(7.27 \pm 0.36) \times 10^{11}\end{array}$ & $\begin{array}{l}02-2007 \\
02-2007 \\
02-2007\end{array}$ & $\begin{array}{l}(2.82 \pm 0.38) \times 10^{12} \\
(3.25 \pm 0.57) \times 10^{12} \\
(7.84 \pm 0.28) \times 10^{11}\end{array}$ \\
\hline PKS 1229-02 & $\begin{array}{l}\mathrm{NaI} \\
-0.00013^{V} \\
-0.00010 \\
\mathrm{Mg} \text { II } \\
0.76836 \\
\mathrm{Mg} \mathrm{II} \\
0.83032^{v} \\
\end{array}$ & $\begin{array}{l}02-2002 \\
02-2002 \\
02-2002 \\
02-2002 \\
\end{array}$ & $\begin{array}{c}\text { significant variability }^{b} \\
(1.98 \pm 0.41) \times 10^{11} \\
(1.81 \pm 0.20) \times 10^{11} \\
\text { no variability } \\
(7.15 \pm 0.24) \times 10^{11} \\
\text { marginal variability }^{c} \\
(3.42 \pm 0.07) \times 10^{12}\end{array}$ & $\begin{array}{l}04-2011 \\
04-2011 \\
04-2011 \\
04-2011 \\
\end{array}$ & $\begin{array}{c}(4.6 \pm 1.5) \times 10^{10} \\
(1.67 \pm 0.16) \times 10^{11} \\
(7.57 \pm 0.58) \times 10^{11} \\
(2.87 \pm 0.13) \times 10^{12}\end{array}$ \\
\hline FBQS J2340-0053 & $\begin{array}{l}\mathrm{C} \text { I } \\
2.05470 \\
2.05473^{v} \\
\mathrm{H}_{2}, J=3 \\
2.05499 \\
2.05513^{v}\end{array}$ & $\begin{array}{l}\text { (blend) } \\
08-2006 \\
08-2006\end{array}$ & $\begin{array}{c}\text { marginal variability }^{d} \\
(1.33 \pm 0.16) \times 10^{13} \\
(3.13 \pm 0.49) \times 10^{13} \\
\text { marginal variability }^{d} \\
(1.66 \pm 0.10) \times 10^{15} \\
(6.19 \pm 0.50) \times 10^{14}\end{array}$ & $\begin{array}{l}10-2008 \\
10-2008\end{array}$ & $\begin{array}{l}(1.25 \pm 0.07) \times 10^{13} \\
(1.52 \pm 0.23) \times 10^{13} \\
(1.43 \pm 0.09) \times 10^{15} \\
(4.39 \pm 0.31) \times 10^{14}\end{array}$ \\
\hline
\end{tabular}

Notes. ${ }^{(a, b, c, d)}$ See Figs. $2-5$ (left panel), 3 (right panel). ${ }^{(V)}$ Significant variability: significance level $>3.5 \sigma$ and $\Delta N /\langle N\rangle>25 \%$. ${ }^{(v)}$ Marginal variability: significance level either in the range $2.0-3.5 \sigma$ or $>3.5 \sigma$ but $\Delta N /\langle N\rangle<25 \%$.

it has nearly disappeared, with $N(\mathrm{Na} \mathrm{I})$ decreasing by a factor of four (see Table 3 and Fig. 2). The three other strong components $\left(N(\mathrm{NaI})>10^{11} \mathrm{~cm}^{-2}\right)$ did not vary $(1 \sigma: 17 \%)$.

Towards PKS 1741-038, Galactic molecular absorptions at $v_{\mathrm{LSR}}=2 \mathrm{~km} \mathrm{~s}^{-1}$ have been extensively investigated with detections of $\mathrm{HCO}^{+}$(Lucas \& Liszt 1996), CO (Akeson \& Blitz 1999), and $\mathrm{C}_{2} \mathrm{H}$ (Lucas \& Liszt 2000), together with variable $21 \mathrm{~cm}$ absorption (Lazio et al. 2001). The associated $\mathrm{Na}$ I absorption at $z_{\mathrm{abs}}=-0.00033$ is multiple (4 components), partly saturated, and blended with atmospheric $\mathrm{Na}$ I emission, thus preventing a search for variations. Two well-known 5780 and $5797 \AA$ DIBs are detected at both epochs. Their equivalent widths are equal to 0.33 and $0.10 \AA$, respectively, and are similar at two epochs $9.8 \mathrm{yr}$ apart with $\Delta W /\langle W\rangle=21 \% \pm 13 \%$ and $20 \% \pm 23 \%$. Two other DIBs at 4428 and $6196 \AA$, the Ca II doublet and $\mathrm{CH}^{+} \lambda 4232$, are detected but are far too noisy in the 2001 spectrum to investigate variability.

Finally, towards FBQS J2340-0053, the NaI 5897 line is not covered in the HIRES spectrum. The single component of the Ca II doublet at $z_{\mathrm{abs}}=-0.00002$ remains stable $(1 \sigma: 3.8 \%)$ within $1.9 \mathrm{yr}$.

\subsection{Neutral and molecular gas at high z}

Towards PKS 0458-020, C I is well detected at $z_{\mathrm{abs}}=1.5605$ in the HIRES spectrum (with a single component, as reported in Kanekar et al. 2010) but with too low a S/N in the UVES spectrum to get useful variability constraints. In FBQS J2340-0053 spectra, $\mathrm{CI}$ is detected at $z_{\mathrm{abs}}=1.3606$ and 2.0545. The three well-separated components of the lower $z$ system are stable $(1 \sigma$ : $12 \%)$. The fit of C I at $z_{\mathrm{abs}}=2.0545$ involves a minimum of eight components, two sets being heavily blended (components 3-4 and 5-6, respectively). Within the blend at $z_{\mathrm{abs}}=2.05473$, a very narrow component (number 6) shows a possible variation $\Delta N /\langle N\rangle$ of $69 \%$ in $0.71 \mathrm{yr}$ (rest frame) at the $3.0 \sigma$ significance level (see Table 3 and Fig. 3 left panel); the $1 \sigma$ limits for the other seven components are in the range $9-50 \%$.
Molecular hydrogen has already been detected towards FBQS J2340-0053 at $z_{\text {abs }}=2.0545$ (Jorgenson et al. 2010). The Lyman and Werner transitions display six components (which roughly correspond to C I components number 1, 2, 3-4, 5-6, 7, and 8), and there is a possible variation of the sixth $\mathrm{H}_{2}, J=3$ weak component at $z_{\mathrm{abs}}=2.05513$. Using the unblended components of the transitions at 1081,1084 , and $1099 \AA$, we find a decrease, $\Delta N /\langle N\rangle=-34 \%$, in only $0.71 \mathrm{yr}$ (rest frame), significant at the $3.1 \sigma$ level (Table 3 and Fig. 3, right panel). Unfortunately, the corresponding $\mathrm{C}$ I features are too weak to check whether C I lines display similar variations (in the left panel of Fig. 3, features at $v \simeq 40 \mathrm{~km} \mathrm{~s}^{-1}$ for the C $\mathrm{I} \lambda 1277$ and $\mathrm{C} \mathrm{I} \lambda 1328$ transitions are in fact dominated by $\mathrm{CI}^{*}$ transitions from the strongest components). Conversely, the fourth $\mathrm{H}_{2}$ component, which corresponds to the second $\mathrm{C} I$ blend (components 5 and 6) with marginal variations is opaque in the available transitions, hence insensitive to time changes.

\subsection{The Mg II-Fe II systems}

\subsubsection{Low $\beta \mathrm{Mg}$ II systems}

There are only two blazars with low $\beta \mathrm{Mg}$ II systems of moderate strength. For the other three, these systems are either DLAs or extremely strong saturated Mg II absorbers. We then searched for proxies of intermediate opacity (e.g. Fe II lines) to constrain variability.

Towards AO $0235+164$, there are three $\mathrm{Mg}$ II systems at low $\beta$ : $z_{\mathrm{abs}}=0.8514$ (three fitted components), 0.8523 (seven components), and 0.85562 (single). There is no variation in the triple and single systems $(1 \sigma$ limits on $\Delta N /\langle N\rangle$ of $9.5 \%$ and $6.3 \%$, respectively). For the multiple system, one Mg II component at $z_{\mathrm{abs}}=0.85236$, blended with two weaker ones at $z_{\mathrm{abs}}=$ 0.85231 and 0.85241 , shows a tentative variability of its column density $\Delta N /\langle N\rangle$ of $53 \%$ in $5.0 \mathrm{yr}$ (absorber rest frame), but at a low significance level of only $2.3 \sigma$ (see Table 3 and 

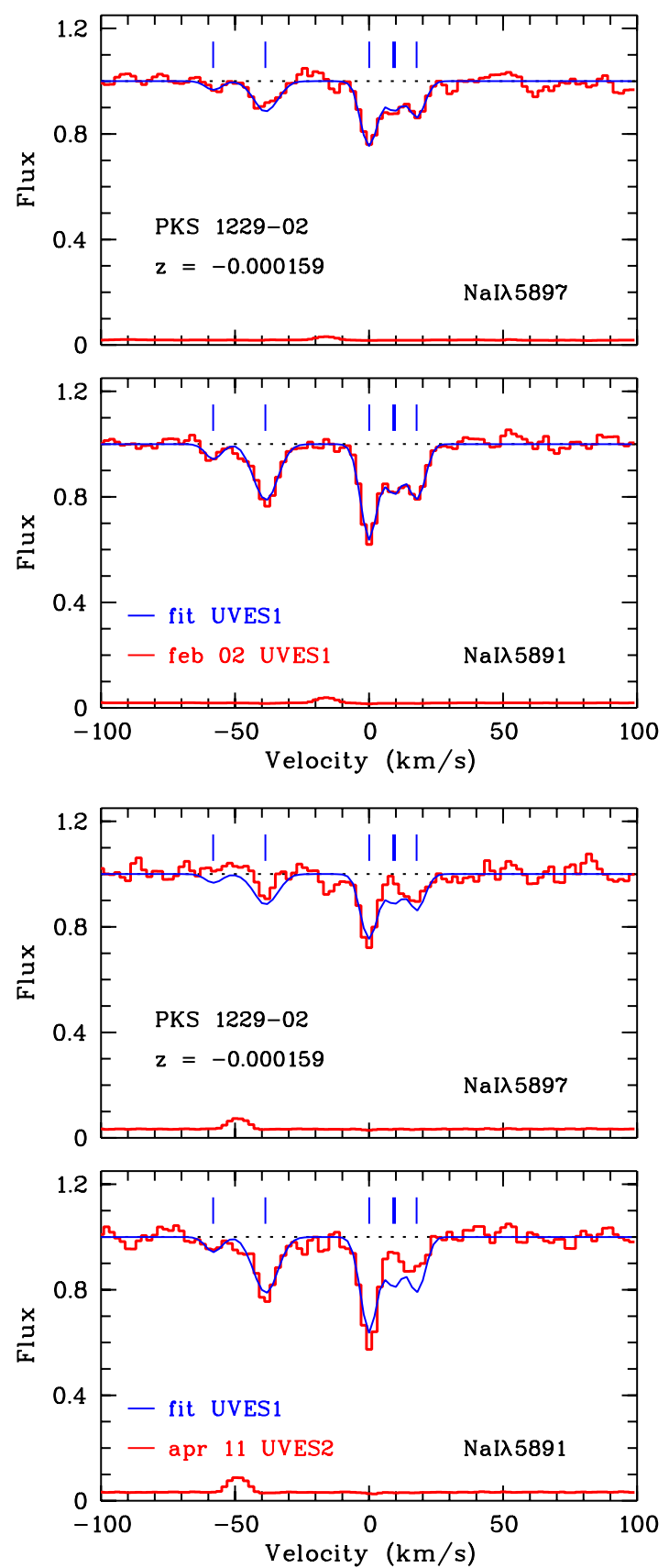

Fig. 2. Galactic Na I absorption toward PKS 1229-02: spectrum, its error (red curves) and simultaneous fit to both transitions (blue curve). The UVES 2002 data are shown in the top panel; in the bottom panel we display the UVES 2011 data together with the fits to the 2002 data (blue). Note the disappearance of the component at $v_{\text {helio }}=9.6 \mathrm{~km} \mathrm{~s}^{-1}$ (thick vertical tick mark), within a period of $9.2 \mathrm{yr}$. The heliocentric redshift $z=-0.000159$ (strongest component) corresponds to $v=0 \mathrm{~km} \mathrm{~s}^{-1}$.

Fig. 4). For these three components, the associated Fe II doublet is stable $(1 \sigma: 5.2 \%)$

Towards PKS 1229-02, there is a triple, unblended system at $z_{\mathrm{abs}}=0.830$. The strongest component at $z_{\mathrm{abs}}=0.83032$ shows a marginal small variation $\Delta N /\langle N\rangle$ of $17 \%$ in $5.0 \mathrm{yr}$ (rest frame) at a $3.7 \sigma$ significance level (Table 3 and Fig. 5). There is no variation in the associated four strongest Fe II lines $(1 \sigma: 7.3 \%)$. The systems at $z_{\mathrm{abs}}=0.83102$ and 0.83128 are too weak to derive meaningful limits on their variabilty. There are two other $\mathrm{Mg}$ II systems at $\beta \leq 0.15$, and they are stable: the weak, single-component doublet at $z_{\mathrm{abs}}=0.7684(1 \sigma: 8.7 \%)$ and the triple system at $z_{\mathrm{abs}}=0.7564$ with one nearly saturated component ( $1 \sigma: 20 \%)$.

Only one of the three targets with extremely strong saturated, low $\beta \mathrm{Mg}$ II systems has proxy lines that yield meaningful limits. Towards PKS 0458-020, the Ni II triplet and the Zn IICrII lines at $z_{\mathrm{abs}}=2.03956$ all have moderate strength and are heavily blended without clear variation. In PKS 1741-038 spectra, the Fe II lines are saturated. Thus, some variability constraint can only be placed on the $z_{\mathrm{abs}}=2.0545$ DLA absorber towards FBQS J2340-0053: the three strongest S II components of medium strength at $z_{\mathrm{abs}}=2.0546$ and the strongest one of $\mathrm{Al}$ III at $z_{\mathrm{abs}}=2.05511$ do not show variability $(1 \sigma: 32 \%$ and $28 \%$, respectively; see Sect. 4.2).

\subsubsection{High $\beta \mathrm{Mg}$ II systems}

For the high $\beta$ systems, variations are not detected from either their $\mathrm{Mg}$ II doublets or proxy lines. Towards AO $0235+164$, the two strongest components of the Ca II doublet at $z_{\text {abs }}=0.5239$, associated with the DLA, do not vary $(1 \sigma: 26 \%)$. Towards PKS 0458-020, the Fe II $\lambda \lambda 2344,2382$ lines at $z_{\mathrm{abs}}=1.5271$ (selecting the 3 unblended components) do not vary either ( $1 \sigma$ : 15\%). Finally, towards FBQS J2340-0053, the highly multiple Fe II system at $z_{\mathrm{abs}}=1.3606$, associated with C I and $21 \mathrm{~cm}$ absorptions, is stable $(1 \sigma: 14 \%$ for all the components, using the weak Fe II $\lambda \lambda 2249,2260$ doublet to constrain the other nearly saturated lines).

\section{Discussion}

\subsection{Presence of intrinsic systems in blazar spectra?}

Time variability of narrow absorption lines at low $\beta$ have been securely detected in small samples of high ionisation systems (involving $\mathrm{CIV}, \mathrm{N} \mathrm{V}$, and $\mathrm{O}$ VI), observed at low and intermediate resolution (Narayanan et al. 2004; Wise et al. 2004; Hamann et al. 2011). The lines showing variations are essentially from mini-BALs and associated $\left(\Delta v<5000 \mathrm{~km} \mathrm{~s}^{-1}\right)$ narrow-line systems. The time-scales investigated (in the quasar rest frame) range from 0.28 to $8.8 \mathrm{yr}$, and the variable narrow lines remained fixed in velocity. High and low ionization systems (at $\beta>0.01$ ) with repeated observations have been identified by Hacker et al. (2013) in a very large sample of SDSS-DR7 quasar spectra: 33 out of 1084 systems have absorption lines with variations in their equivalent widths. For most of them $W_{\mathrm{r}}>0.3 \AA$ and $\Delta W_{\mathrm{r}} / W_{\mathrm{r}}>50 \%$, with two-thirds of the variable systems being at "relatively low" $\beta(<0.22)$. Low ionisation species are present in all the 33 variable systems, and C IV is detected in the 17 systems at sufficiently high $z_{\text {abs }}(>1.50)$. Chen et al. (2013) focussed their variability study on associated $(\beta<0.03) \mathrm{Mg}$ II systems identified in SDSS-DR7 and SDSS-DR9 (BOSS survey) spectra. They do not detect time variations at a significance level higher than $3 \sigma$ in their sample of $36 \mathrm{Mg}$ II doublets.

No variation of intervening Mg II systems in high spectral resolution spectra has been reported so far. Amongst the five targets in our sample, three blazars have in total six Mg II doublets at $\beta \leq 0.15$ that are suitable (unsaturated, not heavily blended lines) for variability studies. As described in Sect. 3.4, only marginal column density variations have been detected for two $z_{\text {abs }} \sim 0.85 \mathrm{Mg}$ II absorbers at significance levels of $2.3 \sigma$ and $3.7 \sigma$, respectively. The four other $\mathrm{Mg}$ II absorbers at $z_{\mathrm{abs}} \sim$ $0.75-0.85$ are stable with $\Delta N /\langle N\rangle$ in the range $6-20 \%$ at $1 \sigma$ confidence level. Among them, the one in the spectrum of 

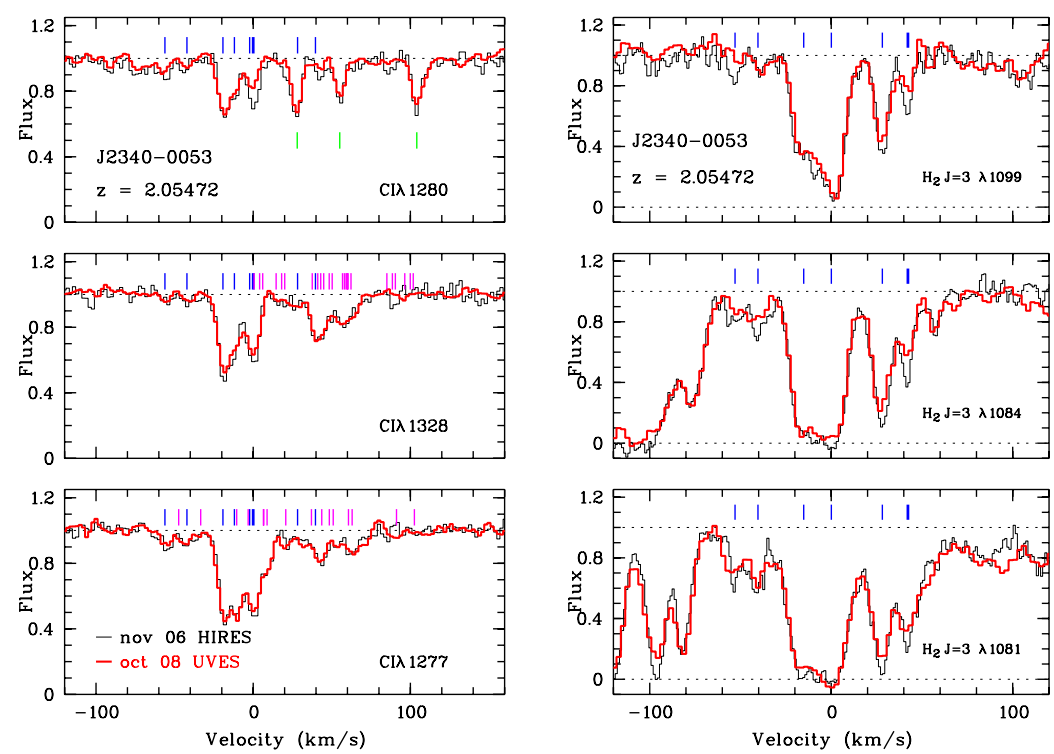

Fig. 3. C I $\lambda 1277,1280,1328$ and molecular $\mathrm{H}_{2}, J=3 \lambda 1081,1084,1081$ absorptions at $z_{\text {abs }}=2.05472$ towards FBQS J2340-0053 in the November 2006 (HIRES, black line) and October 2008 (UVES, red line) spectra. Left panel: the eight C I components required to fit the profiles are shown by blue ticks, and the marginally varying feature is at $v=0$ (thick tick). In the top C I $\lambda 1280$ plot, three $\mathrm{C} \mathrm{I} \lambda 1656$ lines from the $z_{\text {abs }}=1.3606$ system are also shown (green ticks). For the $\mathrm{C} \mathrm{I} \lambda 1277$ and $\mathrm{C} \mathrm{I} \lambda 1328$ transitions, we show the position expected for $\mathrm{C} \mathrm{I}^{*}$ transitions associated with the 8 components (cyan ticks). The features at $v \simeq 40 \mathrm{~km} \mathrm{~s}^{-1}$ are mainly due to $\mathrm{CI}^{*}$ transitions. Right panel: six $\mathrm{H}_{2} \mathrm{~J}=3 \mathrm{com}$ ponents are required to fit the profiles. Marginal variability is seen in the sixth component (thick tick) at $v \simeq 40 \mathrm{~km} \mathrm{~s}^{-1}$. Some difference is also seen in the fifth component, but it is significant only at the $2.0 \sigma$ level, instead of $3.1 \sigma$ for the sixth one. For both narrow components displaying marginal variations in $\mathrm{CI}(v \simeq 0)$ or $\mathrm{H}_{2}\left(v \simeq 40 \mathrm{~km} \mathrm{~s}^{-1}\right)$, we checked that the slightly higher resolution of the HIRES spectrum cannot account for the observed difference between the profiles.
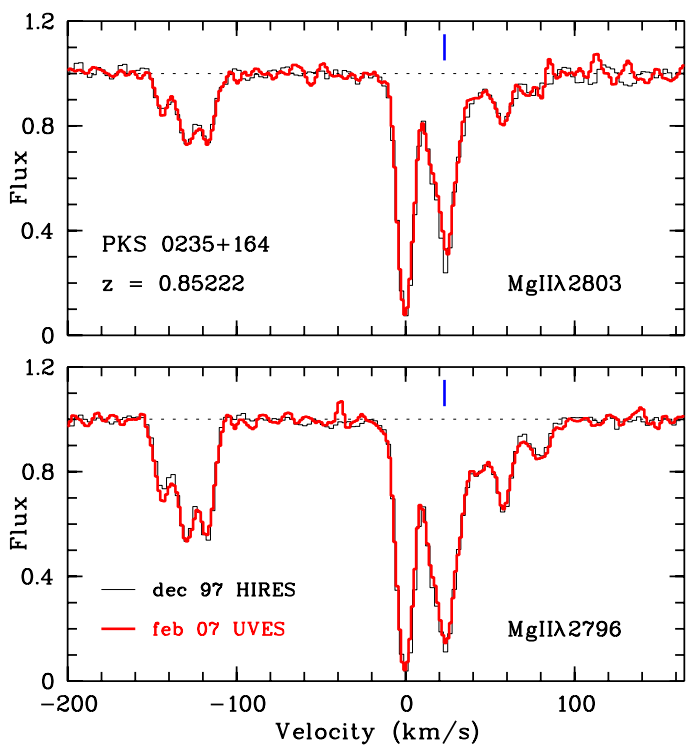

Fig. 4. Intervening $\mathrm{Mg}$ II absorption at $z_{\mathrm{abs}}=0.852$ towards AO $0235+164$ in the 1997 December (HIRES, black line) and 2007 February (UVES, red line) spectra (lower panel: Mg II $\lambda 2796$; upper panel: $\mathrm{Mg}$ II $\lambda 2803)$. The blue vertical tick underlines a marginally variable blended component at $z_{\text {abs }}=0.85237$, although at only a $2.3 \sigma$ significance level.

AO $0235+164$ is $150 \mathrm{~km} \mathrm{~s}^{-1}$ away from the tentatively variable system.

In Bergeron et al. (2011), we found a potential excess of Mg II systems at $\beta \approx 0.1$ in blazar spectra and proposed that gas swept up by the AGN jet could be responsible for it. In such a scenario, absorption line variability can be expected, but, in the absence of predictions from numerical simulations, it is difficult to anticipate the time scale and magnitude expected for the column density changes; this should depend strongly on the occurrence of marked variations in the activity of the nucleus itself at about the time when the blazar was observed. Thus, we cannot really draw conclusions from the lack of clear evidence for time variations among the limited set of systems that we could investigate here.

\subsection{Structure in moderately ionised halo gas}

We now discuss systems involving narrow absorption lines associated with halo gas that is optically thick to UV ionizing radiation, i.e. gas classically traced by $\mathrm{Mg}$ II or Fe II transitions. We mentioned above the few cases for which we could get stringent upper limits on column density variations for discrete velocity components seen through intermediate opacity lines. One good example is the $z_{\mathrm{abs}}=1.3606$ system towards FBQS J2340-0053 involving 10 Fe II components. Even when most of the absorption profile is optically thick (this is often the case for the Mg II doublet), the data can be used to search for variations near the profile edges (e.g. appearance or disappearance of faint components). We could not identify any change of this kind. Moreover, since the component redshifts are allowed to be free when fitting the profiles, we could also get constraints on velocity variations; again, we found no significant change, with $3 \sigma$ upper limits generally below $1 \mathrm{~km} \mathrm{~s}^{-1}$. Occasionally, some line profiles are observed to be rather smooth as seen for the resolved S II $\lambda 1253$ and 1259 lines at $z_{\mathrm{abs}}=2.05454$ in the FBQS J2340-0053 spectrum. As shown in Fig. 6, no significant difference is seen along the entire profile. The $1 \sigma$ upper limit on the relative flux variation is $1.7 \times 10^{-2}$ per resolution element along the S II $\lambda 1259$ line profile. Since the latter spans over $120 \mathrm{~km} \mathrm{~s}^{-1}$, the previous upper limit applies to about $120 / 6.6 \simeq 18$ distinct resolution elements. 
P. Boissé et al.: Absorption line variations in quasar spectra
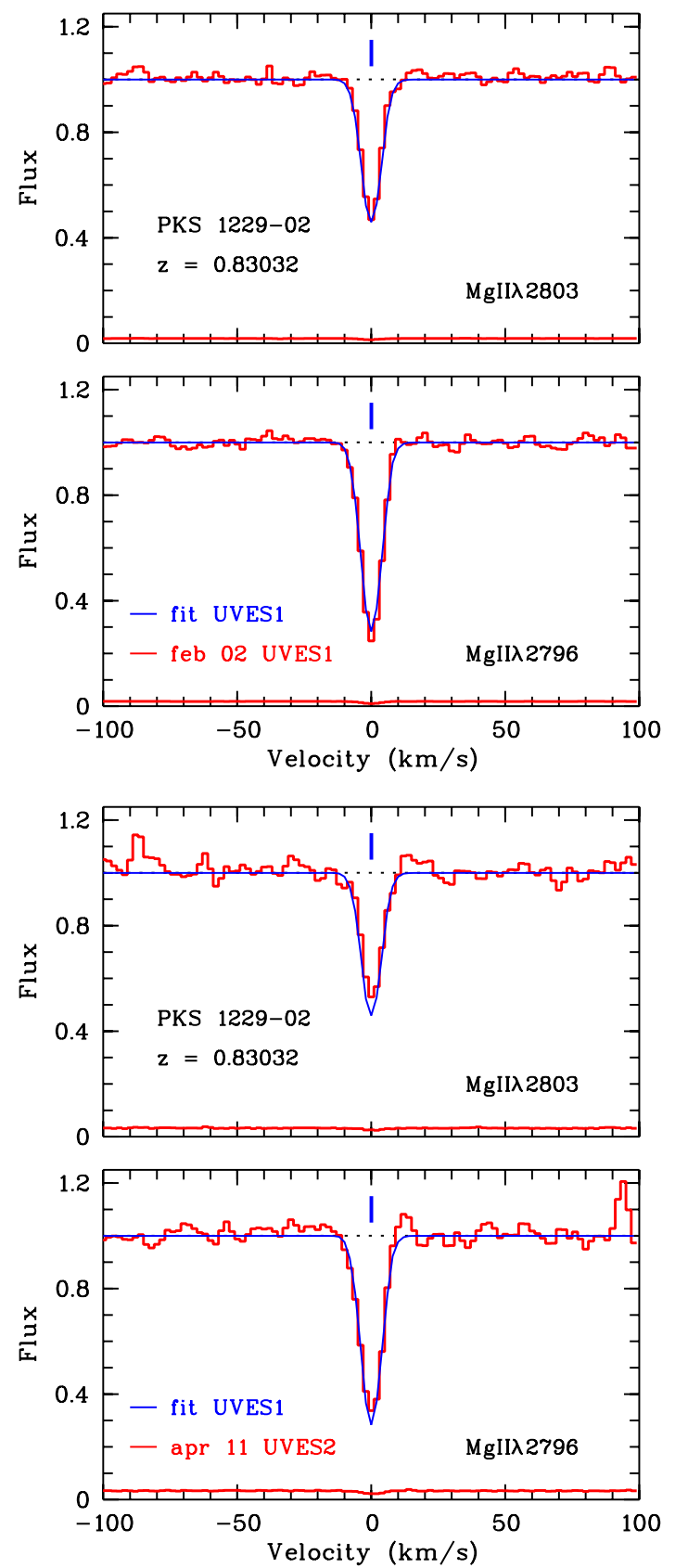

Fig. 5. Intervening $\mathrm{Mg}$ II absorption at $z_{\mathrm{abs}}=0.83032$ towards PKS 1229-02: spectrum and its error (red curves), together with a simultaneous fit to both transitions (blue curve). In the upper panel, we show the UVES 2002 data, while the bottom panel displays the UVES 2011 data with the fit performed on the UVES 2002 spectrum. The blue vertical tick underlines the marginally variable Mg II doublet.

To conclude the discussion for these systems, we detected no structure in the density and velocity field within $\mathrm{Mg}$ II/Fe II halos on the scales that we could probe (typically of the order of $100 \mathrm{au}$ ). This is consistent with previous observations of moderately ionised gas in galaxy halos. Indeed, both Rauch et al. (2002) and Kobayashi et al. (2002) find that a typical value for the cloud size associated with the individual velocity components is $200 \mathrm{pc}$, implying very little structure on the much smaller scales that we probe here. We also note that, from the analysis of the number density of strong Mg II systems detected on top of quasar emission lines in SDSS spectra, Lawther et al. (2012) derive a lower limit of about $0.03 \mathrm{pc}$ for the individual
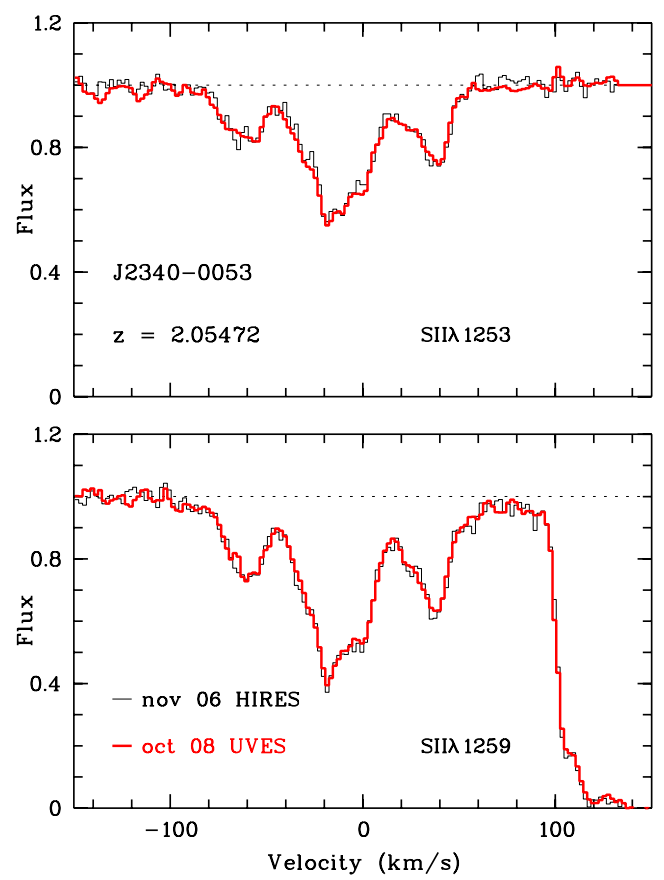

Fig. 6. S II $\lambda \lambda 1253,1259$ absorption at $z_{\text {abs }}=2.05454$ towards FBQS J2340-0053 in the 2006 November (HIRES, black line) and 2008 October (UVES, red line) spectra.

Mg II absorbing clouds. Finally, Welty (2007) analysed multiepoch HST spectra of HD 219188 and detect no variations for S II, O I, Si II, and Fe II in the disk of our own Galaxy, on scales of the order of $100 \mathrm{au}$. Thus, our results are in good agreement with all known constraints on structure in moderately ionised gas.

In this context, the variations tentatively detected by Hacker et al. (2013) for narrow intervening Mg II systems are most surprising. These authors analysed more than 1000 reliable quasar absorption systems with multi-epoch observations and extracted a small subset of 33 systems displaying some evidence for time changes in their equivalent widths. Their study differs markedly from ours in the number of systems investigated, and given the small fraction of systems showing variability - about $3 \%$ - it is true that we expect very few variable lines in our sample. However, it should be stressed that the two studies also differ strongly in the sensitivity expressed in terms of $\Delta W_{\mathrm{r}}$ or $\Delta W_{\mathrm{r}} / W_{\mathrm{r}}$. The variations reported in the SDSS spectra are very large with, in most cases, $\Delta W_{\mathrm{r}}>0.3 \AA$ and $\Delta W_{\mathrm{r}} / W_{\mathrm{r}}>50 \%$, corresponding to even greater fractional column density variations. For comparison, the marginal variation quoted above in the $\mathrm{Mg}$ II $\lambda 2796$ line at $z_{\mathrm{abs}}=0.83032$ towards PKS $1229-02$ is smaller than $\Delta W_{\mathrm{r}}=0.01 \AA$ and $\Delta W_{\mathrm{r}} / W_{\mathrm{r}}=5 \%$.

If the large variations quoted by Hacker et al. (2013) are real, one can imagine that the number of systems expected to display changes at the level that we can reach in our high resolution study should be much greater, coming into contradiction with the absence of clear detection of variations in our sample. Before drawing any conclusion, additional observations aiming at confirming the status of the 33 potentially variable systems would be highly desirable. In particular, since variations are (to first order) expected to be regular, a third spectrum should confirm the trend observed on the two first ones. Such a work is currently in progress (see Appendix B2 in Dawson et al. 2013). Repeated high resolution observations of the brightest quasars 
involved would also be very useful for checking the reality of time variations and characterising them.

\subsection{High z neutral/molecular gas}

$\mathrm{C} \mathrm{I}$ and $\mathrm{H}_{2}$ are known to be closely related in diffuse molecular clouds (Srianand et al. 2005). Indeed, the velocity components found for both species are generally similar, although some significant differences in their relative strength can be observed, as is the case for the $z_{\mathrm{abs}}=2.0545$ system towards FBQS J2340-0053 (Sect. 3.3 and Fig. 3). We successively discuss these two tracers below.

$\mathrm{C}$ I lines are detected in three high $z$ systems, one towards PKS 0458-020 and two towards FBQS J2340-0053, but useful constraints could be obtained only for the last two at $z_{\text {abs }}=$ 1.3606 (no change in $N$ ) and 2.0454 (possible variation of about $70 \%$ for an unresolved component). Unfortunately, since i) the transverse velocity of the quasar is unknown; and ii) the LoS drift is determined solely by the quasar motion for the $z_{\mathrm{abs}}=2.0545$ system because $z_{\mathrm{abs}} \simeq z_{\mathrm{em}}$, it is not possible to compute the scale probed through the neutral gas. Furthermore, since the transverse observer's velocity for this target is only $12 \mathrm{~km} \mathrm{~s}^{-1}$, little drift due to the observer's motion is expected for the $z_{\mathrm{abs}}=1.3606$ absorber. We can then just get a typical value for the drifts by assuming a transverse velocity of about $300 \mathrm{~km} \mathrm{~s}^{-1}$ for the intervening galaxies. This value, together with a time interval of $1.9 \mathrm{yr}$ (observer's frame) between the two observations, corresponds to a linear scale of about 50 and 40 au at $z_{\text {abs }}=1.3606$ and 2.0545 , respectively. In our own Galaxy, the available information on structure in the C I distribution remains quite limited. Welty (2007) performed a detailed study of the time behaviour observed for visible and UV transitions due to an intermediate velocity cloud towards the star HD 219188. Variations by a factor of about two are seen for $\mathrm{NaI}, \mathrm{CI}$, and $\mathrm{Ca}$ II column densities on scales of tens of au. Qualitatively, Na I and CI display the same time behaviour, indicating that the more extensive results obtained on the Na I structure (Crawford 2003; Smoker et al. 2011) are also probably valid for $\mathrm{C}$ I. In this context, results obtained for $N(\mathrm{C} \mathrm{I})$ at $z_{\mathrm{abs}}=1.3606$ and 2.0545 are certainly consistent with the properties known for Galactic gas. Clearly, studies involving more systems are desirable to reach firm conclusions.

Regarding $\mathrm{H}_{2}$, velocity components number 5 and 6 display an intermediate opacity that provides good sensitivity for the $J=3$ column densities. The other $J=3$ components are either too weak or too saturated; similarly, $J=2$ lines are too strong, while $J=4$ are too weak to be suitable. The detection of marginal variations in component number 6 is interesting in the context of questions related to the "warm $\mathrm{H}_{2}$ " observed along many Galactic LoS (Gry et al. 2002; Jensen et al. 2010). Ultraviolet pumping is generally found to be insufficient for explaining the observed excess of $J \geq 2 \mathrm{H}_{2}$ with respect to the amount expected for a thermal distribution (Gry et al. 2002). Small structures (like shocks or turbulent vortices) where the temperature can locally reach high values have been proposed to account for both the production of species like $\mathrm{CH}^{+}$and the relative amount of $J \geq 2 \mathrm{H}_{2}$ (Godard et al. 2009). Presently, there are no direct constraints on the size of these putative regions.

In our Galaxy, the only repeated measurements in the far-UV were performed towards the runaway O star, HD 34078 (Boissé et al. 2009). Stringent constraints were derived from the analysis of damped line profiles associated with $J=0$ lines, but no limit could be obtained for the $J=2,3,4$ levels because the corresponding lines are insensitive to small relative changes in $N$. It is noticeable that for the varying component towards FBQS J2340-0053, the $N(J=0,1,2,3)$ values $\left(1.39 \times 10^{17}\right.$, $2.61 \times 10^{17}, 4.71 \times 10^{15}$ and $5.28 \times 10^{14} \mathrm{~cm}^{-2}$; for the $J=3 \mathrm{col}$ umn density, we give the average of the 2006 and 2008 values) are consistent with a unique excitation temperature, $T_{\mathrm{ex}}=$ $120 \mathrm{~K}$, unlike what is seen over most of Galactic LoS where an excitation temperature of about $300 \mathrm{~K}$ is required to fit $N(J)$ values at $J \geq 2$ (Jensen et al. 2010). This indicates that the gas is dense enough to ensure thermalisation, which is in qualitative agreement with the small size suggested by the time variations observed for this component.

\subsection{Local Galactic gas}

We found a large variation (factor of four) in Galatic N(Na I) for one of the five velocity components towards PKS 1229-02. The constraint on the corresponding cloudlet size can be estimated from the peculiar motion of the Sun with respect to the LSR (see Sect. 2.2). For this direction, the Sun's velocity is mostly transverse with $V_{\mathrm{t}}(\mathrm{Sun}) / \mathrm{LSR}=17.8 \mathrm{~km} \mathrm{~s}^{-1}$, implying a drift of about $35 \mathrm{au}$. This is well within the range (typically a few $10 \mathrm{au}$ or larger) for which $\mathrm{NaI}$ line changes have been observed on other LoS (Crawford 2003). The other four components towards PKS 1229-02 remained stable on the same linear scale, indicating that the associated fragments are characterised by sizes larger than $100 \mathrm{au}$. For other targets providing useful constraints on Galactic gas structure, we find variation neither towards AO 0235+164 nor towards FBQS J2340-0053. The transverse velocity of the Sun for these two targets is $15.8 \mathrm{~km} \mathrm{~s}^{-1}$ and $16.6 \mathrm{~km} \mathrm{~s}^{-1}$, respectively, implying drifts of about 30 and 7 au in local material. In these two cases, $\mathrm{Na}$ I lines have a relatively high opacity, implying a poor sensitivity to variations.

Towards PKS 1741-038, the estimated drift over the $9.8 \mathrm{yr}$ separating the two observations is about 17 au. The absence of variations in DIBs near 5780.5 and $5797.1 \AA$ can be related to results obtained recently by Cordiner et al. (2013) for $\rho$ Oph stars. These authors find variations of about 5-9\% for the above DIBs on scales exceeding 300 au. Thus, given our limited sensitivity, the observed stability is not surprising.

\section{Summary and prospects for further studies}

As discussed above, the only clear and unambiguous variation has been detected for the Na I doublet from our own Galaxy towards PKS 1229-02, in good agreement with previous results on the au-scale structure of neutral Galactic gas. In the same kind of media but at higher redshifts ( $z \approx 2$ toward FBQS J2340-0053), we also find tentative evidence of structure in neutral and molecular gas: C I with $\Delta N /\langle N\rangle \simeq 70 \%$ at the $3.0 \sigma$ significance level and $\mathrm{H}_{2}$ with $\Delta N /\langle N\rangle \simeq 35 \%$ at the $3.1 \sigma$ level. For low $\beta$ absorption systems in blazar spectra, absorption profiles are, for the most part, very stable and only a marginal $3.7 \sigma 17 \%$ variation has been seen in the strength of a narrow $\mathrm{Mg}$ II component towards PKS 1229-02. On the other hand, no change was seen for moderately ionised gas at high $\beta$ (i.e. intervening halo gas), which again is consistent with known constraints on typical cloud sizes in such media.

The results discussed above illustrate well the potential and the limits of the method used in this paper to investigate the small-scale structure of absorbing gas in quasar spectra. We first outline the high value of high spectral resolution. Often, several transitions from the same species are detected and can be used to fit absorption line profiles, thus yielding well constrained $b$ and 
$N$ values for the velocity components seen at each epoch. This redundancy provides a powerful objective way (at least for those components that are partially resolved) to assess the reality of time changes and rule out false variations. Indeed, it is very unlikely to find two artefacts mimicking true variations by chance, i.e. occurring on two line profiles at the same position in velocity space and with a strength consistent with the $f$ values of the transitions involved. If species displaying more than two transitions (like $\mathrm{C}$ I or $\mathrm{H}_{2}$ ) can be used to increase the redundancy and the range covered by $f$ values, the probability is even lower, and better constraints can be derived from line fitting. Conversely, the $\mathrm{Mg}$ II system at $z_{\mathrm{abs}}=0.85236$ towards $\mathrm{AO} 0235+164$ illustrates well that with only two transitions differing in their $f$ values by a factor no higher than two, one may be unable to reach firm conclusions when variations are moderate. Furthermore, high resolution allows us to quantify the changes in meaningful astrophysical parameters, $N$ and $b$ values, or to set upper bounds on their variations. Limitations appear, however, for some line profiles (e.g. the C I component towards FBQS J2340-0053 for which variations are suspected), when the decomposition into discrete components required by line fitting is poorly defined or not unique. Such difficulties mean that the spectral resolution used is not high enough to analyse the velocity distribution properly, implying that line opacities, as well as $N$ and $b$ values, cannot be determined reliably. One should therefore prefer absorption systems with simple velocity structure for repeated observations.

Our study considered several topics for which useful constraints can be obtained from an investigation of time variations. We now discuss prospects for two of them. We mentioned above that, generally, we have poor knowledge of the scales probed by repeat observations because the transverse motion of the target is not measurable. The situation is more favourable for low redshift absorbers because the drift of the LoS through the intervening gas is determined mainly by the observer's motion, which is inferred directly from observations of the CMB dipole. For instance, to study whether the small-scale structure seen in the Galactic neutral or weakly ionised gas is ubiquitous in external galaxies, one could observe quasars belonging to the socalled quasar-galaxy pairs again (see e.g. Bowen et al. 1991) and search for temporal changes in the $\mathrm{Ca}$ II or $\mathrm{NaI}$ lines over more than $20 \mathrm{yr}$. Of course, targets located in directions that are more or less normal to our large-scale peculiar velocity should be preferred (in this favourable configuration, the scales probed over $20 \mathrm{yr}$ are of the order of $1500 \mathrm{au}$ ). Regarding high redshift absorbers, for which only a rough estimate of the scales probed can be obtained, one needs to observe a statistically significant sample of targets to derive meaningful results concerning the presence and strength of small-scale structure. Some quasars are found in pairs (Hennawi et al. 2010), and occasionally, absorbers appear to belong to galaxy clusters (Whiting et al. 2006), implying increased peculiar velocities. Such LoS will, on average, be associated with larger drifts, and should be targetted preferentially.

A second topic for which repeated observations of quasars at high spectral resolution are especially timely involves the properties and origin of the warm $(J \geq 2) \mathrm{H}_{2}$ found in diffuse molecular gas in our own Galaxy, as well as in high redshift absorbers. During the past ten years, the number of high $z$ $\mathrm{H}_{2}$ systems has increased very significantly and now exceeds 20 (Albornoz-Vásquez et al. 2014). Some of them display narrow velocity components that are well suited to a detailed study (see e.g. Noterdaeme et al. 2007). As discussed in Sect. 3 for the system at $z_{\text {abs }}=2.0545$ towards FBQS J2340-0053, multi-epoch observations can provide key information on the typical size of regions where diffuse molecular gas is heated to temperatures above $200 \mathrm{~K}$. Such high $z$ warm $\mathrm{H}_{2}$ is seen at epochs when star formation was much more efficient than today (Madau \& Dickinson 2014), and one can therefore anticipate that the diffuse molecular medium should be strongly affected by a very high dissipation rate of the mechanical energy carried by shock waves and turbulence.

To conclude, we wish to stress that the data used here were acquired for purposes other than variability searches and thus are certainly not the best choice for our study. First, the two spectra available for each target often differ significantly in their $\mathrm{S} / \mathrm{N}$, and one is then limited in the comparison by the lower quality spectrum. The time interval also varies a lot from target to target and, unfortunately, the lag for the quasar displaying the richest spectrum - FBQS J2340-0053 - is of only two years. A straightforward strategy for getting better constraints or addressing some of the questions discussed above with more appropriate data would consist in i) selecting targets that display the required types of absorption lines and targets with one already available good high resolution spectrum taken at least ten years ago; and ii) observing them again at similar $\mathrm{S} / \mathrm{N}$ and resolution. Obviously, the existence of databases where high resolution quasar spectra are archived properly are extremely useful in such an approach. Finally, we note that improvements in the accuracy of wavelength calibrations accomplished recently in the context of the ESO Large Programme designed to constrain the variation in fundamental constants (Molaro et al. 2013) or improvements foreseen for instruments that will become available in the near future (e.g. the ESPRESSO spectrograph to be mounted on the ESO/VLT telescope) should indeed help to investigate the velocity field within the absorbers in more detail.

Acknowledgements. We are very grateful to several colleagues who provided some of the spectra used in our study or contributed to their reduction: John Black (for the 2001 PKS 1741-038 spectrum), Cédric Ledoux (PKS 0458020, UVES data), Pasquier Noterdaeme and Hadi Rahmani (FBQS J2340-0053, UVES data), John O'Meara (AO 0235+164, HIRES spectrum), and Giovanni Vladilo (UVES spectra of AO 0235+164 and PKS 1229-02). Thanks are also due to Jean-Philippe Uzan for discussions about the estimate of line-of-sight drifts on cosmological scales and to Gary Mamon for help on data handling techniques. We are grateful to the referee for several constructive comments that helped to clarify the content of this paper.

\section{References}

Akeson, R. L., \& Blitz, L. 1999, ApJ, 523, 163

Albornoz-Vàsquez, D., Rahmani, H., Noterdaeme, P., et al. 2014, A\&A, 562, A88

Bergeron, J., Petitjean, P., Aracil, B. et al. 2004, The Messenger, 118, 40

Bergeron, J., Boissé, P., \& Ménard, B. 2011, A\&A, 525, A51

Boissé, P., Rollinde, E., Hily-Blant, P., et al. 2009, A\&A, 501, 221

Boissé, P., Federman, S. R., Pineau Des Forêts,

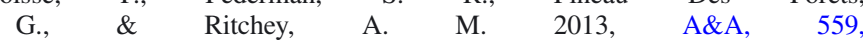

A 131

Bowen, D. V., Pettini, M., Penston, M. V., \& Blades, C. 1991, MNRAS, 249, 145 Burbidge, E. M., Beaver, E. A., Cohen, R. D., Junkkarinen, V. T., \& Lyons, R. W. 1996, AJ, 112, 2533

Chen, Z.-F., Pan, C.-J., Li, G.-Q., Huang, W.-R., \& Li, M.-S. 2013, JA\&A, 34, 317

Churchill, C. W., \& Charlton, J. C. 1999, AJ, 118, 59

Cordiner, M. A., Fossey, S. J., Smith, A. M., \& Sarre, P. J. 2013, ApJ, 764, 10 Crawford, I. A. 2003, Ap\&SS, 285, 661

Crighton, N. H. M., Hennawi, J. F., Simcoe, R. A., et al. 2015, MNRAS, 446, 18 Dai, X., Kochanek, C. S., Chartas, G., et al. 2010, ApJ, 709, 278 Dawson, K. S., Schlegel, D. J., Ahn, C. P., et al. 2013, AJ, 145, 10 Filiz, A.k., N., Brandt, W. N., Hall, P. B., et al. 2013, MNRAS, 777, 168 Fixsen, D. J., Cheng, E. S., Gales, J. M., et al. 1996, ApJ, 473, 576 Godard, B., Falgarone, E., \& Pineau Des Forêts, G. 2009, A\&A, 495, 847 Grier, C. J., Hall, P. B., Brandt, W. N., et al. 2015, ApJ, 806, 111 
Gry, C., Boulanger, F., Nehmé, C., et al. 2002, A\&A, 391, 675

Hacker, T. L., Brunner, R. J., Lundgren, B. F., \& York, D. G. 2013, MNRAS, 434, 183

Hennawi, J. F., Myers, A. D., Shen, Y., et al. 2010, ApJ, 719, 1672

Hamann, F., Kaplan, K. F., Rodriguez Hidalgo, P., Prochaska, J. X., \& Herbert-Fort, S. 2008, MNRAS, 391, L39

Hamann, F., Kanekar, N., Prochaska, J. X., et al. 2011, MNRAS, 410, 1957

Jensen, A. G., Snow, T. P., Sonneborn, G., \& Rachford, B. L. 2010, ApJ, 711, 1236

Jorgenson, R. A., Wolfe, A. M., \& Prochaska, J. X. 2010, ApJ, 723, 460

Kanekar, N., \& Chengalur, J. N. 2001, MNRAS, 325, 631

Kanekar, N., Prochaska, J. X., Ellison, S. L., \& Chengalur, J. N. 2010, ApJ, 712, L152

Kanekar, N., Prochaska, J. X., Smette, A., et al. 2014, MNRAS, 438, 2131

Kobayashi, N., Terada, H., Goto, M., \& Tokunaga, A. 2002, ApJ, 569, 676 Lauroesch, J. T. 2007, ASP Conf. Ser., 365, 40

Lawther, D., Paarup, T., Schmidt, M., et al. 2012, A\&A, 546, A67

Lazio, T. J. W., Gaume, R. A., Claussen, M. J., et al. 2001, ApJ, 546, 267

Le Brun, V., Bergeron, J., Boissé, P., \& Deharveng, J. M. 1997, A\&A, 321, 733

Lucas, R., \& Liszt, H. 1996, A\&A, 307, 252
Lucas, R., \& Liszt, H. 2000, A\&A, 358, 1069

Madau, P., \& Dickinson, M. 2014, ARA\&A, 52, 415

Meyer, D. M., Peek, J. E. G., Peek, J. E. G., \& Heiles, C. 2012, ApJ, 752, 119

Misawa, T., Charlton, J. C., \& Eracleous, M. 2014, ApJ, 792, 77

Molaro, P., Centurión, M., Whitmore, J. B., et al. 2013, A\&A, 555, A68

Narayanan, D., Hamann, F., Barlow, T., et al. 2004, ApJ, 601, 715

Neeleman, M., Prochaska, J. X., \& Wolfe, A. M. 2015, ApJ, 800, 7

Noterdaeme, P., Ledoux, C., Petitjean, P., et al. 2007, A\&A, 474, 393

Rahmani, H., Srianand, R., Gupta, N., et al. 2012, MNRAS, 425, 556

Rauch, M., Sargent, W. L. W., Barlow, T. A., \& Simcoe, R. A. 2002, ApJ, 576, 45

Savage, B. D., \& Sembach, K. R. 1991, ApJ, 379, 245

Srianand, R., Petitjean, P., Ledoux, C., Ferland, G., \& Shaw, G. 2005, MNRAS, 362

Smoker, J. V., Bagnulo, S., Cabanac, R., et al. 2011, MNRAS, 414, 59

Welty, D. E. 2007, ApJ, 668, 1012

Whiting, M. T., Webster, R. L., \& Francis, P. J. 2006, MNRAS, 368, 341

Wise, J. H., Eracleous, M., Charlton, J. C., \& Ganguly, R. 2004, ApJ, 613, 129

Wolfe, A. M., Davis, M. M., \& Briggs, F. H. 1982, ApJ, 259, 495 